\title{
Infrared Asymptotic Freedom for the Pseudoscalar Yukawa Model at the Critical Point
}

\author{
J. Dimock \\ Department of Mathematics, SUNY at Buffalo, Buffalo, NY 14214, USA
}

\begin{abstract}
We consider the pseudoscalar Yukawa model on a four dimensional Euclidean lattice. The fermions are integrated out giving an effective boson interaction. We show that for weak coupling this interaction is in the class covered by the Gawedzki-Kupiainen renormalization group analysis. It follows that one can adjust parameters so that the flow of the renormalization group is toward a free massless boson theory. This establishes the existence of the critical theory and controls its long distance behavior.
\end{abstract}

\section{Introduction}

There has recently been substantial progress made in developing rigorous non-perturbative renormalization schemes for quantum field theory $([1,2,4,7-9,12-14])$. Much of this work uses Wilson's formulation of the renormalization group.

In this approach one begins with a lattice field theory on a fine lattice and attempts to take the continuum limit by studying effective interactions on coarser lattices obtained by block spin transformations. This is the basic short distance or ultraviolet problem. The problem can also be formulated as the problem of studying the scaling limit for a critical field theory on a unit lattice. In this version a first step is obtaining the critical theory, a question which is also of interest for statistical mechanics. Thus one arrives at a class of long-distance or infrared problems which still carry many of the basic problems of renormalization. The technique for controlling the infinite volume limit is again to use block spin transformations, this time to get effective interactions in smaller volumes.

In this paper we study the infrared problem for a critical pseudoscalar Yukawa theory. Our approach uses the renormalization group analysis of Gawedzki and Kupiainen ([10-13]). The remainder of this introduction is devoted to a precise description of their results, and in the balance of the paper we show how they can be applied to our model.

Let $L$ be an odd integer and let $\Lambda$ be a four-dimensional unit toroidal lattice 
with $L^{N}$ points in each direction

$$
\Lambda=\Lambda_{N}=\left(\mathbb{Z} / L^{N} \mathbb{Z}\right)^{4} \cong\left(\left[-L^{N} / 2, L^{N} / 2\right] \cap \mathbb{Z}\right)^{4} .
$$

Fields $\varphi$ on $\Lambda$ are functions $\varphi \in \mathbb{R}^{\Lambda}$. The Hamiltonian $\mathscr{H}_{0}$ consists of a free massless piece and a small perturbation:

$$
\mathscr{H}_{0}(\varphi)=\frac{1}{2} \sum_{x, y \in \Lambda} \varphi_{x}\left(G_{0}^{-1}\right)_{x y} \varphi_{y}+V(\varphi)
$$

Here $G_{0}^{-1}=G_{0}^{-1}(\xi)$ is a version of the lattice Laplacian:

$$
\left(G_{0}^{-1}\right)_{x y}=-\Delta_{x y}+\xi|\Lambda|^{-1} \quad \xi>0 .
$$

The $\xi$ term is added so that the inverse $G_{0}$ exists, but infinite volume quantities will be independent of $\xi$. The potential $V$ is assumed to satisfy $V(0)=0$ and $V(\varphi)=$ $V(-\varphi)$ and to have all the symmetries of the lattice. In addition there are assumptions about the size and locality of $V$ which we explain after some more definitions.

We divide $\Lambda$ up into blocks $\Delta$ with $L^{N_{0}}$ points on a side and centered on the points of $\left(L^{N_{0}} \mathbb{Z}\right)^{4}$. A paved set $Y$ is a union of blocks $\Delta$. For a paved set $Y,|Y|$ is the number of blocks in $Y$ and $\mathscr{L}(Y)$ is defined to be the length of shortest tree graph on the centers of the blocks in $Y$. The potential will have pieces of the form $\sum_{Y \subset A} V_{Y}$, where the sum is over non-empty paved subsets of $\Lambda, V_{Y}$ depends only on $\varphi \uparrow Y$, and $V_{Y}$ is exponentially decreasing in $\mathscr{L}(Y)$.

One distinguishes between large and small field regions. The small field region includes complex-valued fields and has the form $\mathscr{K}(\Lambda)$, where for any paved set $X \subset \Lambda$ :

$$
\begin{aligned}
\mathscr{K}(X)= & \left\{\varphi \in \mathbb{C}^{X}:\left|\varphi_{x}\right| \leqq C_{1} n_{0}^{1 / 4},\left|\varphi_{x}-\varphi_{y}\right| /|x-y|<C_{0} C_{1} n_{0}^{1 / 4},\right. \\
& \left.\left|\left(\partial_{\mu} \varphi\right)_{x}-\left(\partial_{\mu} \varphi\right)_{y}\right| /|(x-y)|^{2 / 3} \leqq C_{0} C_{1} n_{0}^{1 / 4}, x \neq y\right\} .
\end{aligned}
$$

Gawedzki-Kupiainen assume the following condition is satisfied for the small field region:

GKI: $V$ is analytic on $\mathscr{K}(\Lambda)$. In the Taylor series $V=V_{2}+V_{4}+V_{\geqq 6}$ around zero we have

$$
\begin{aligned}
V_{2}(\varphi) & =\sum_{x}\left(\frac{1}{2} m_{0}^{2}-6 \lambda_{0} G_{0, x x}\right) \varphi_{x}^{2}+\sum_{\mu, v x, y} \sum_{\mu}\left(\partial_{\mu} \varphi\right)_{x} K_{\mu v}(x, y)\left(\left(\partial_{v} \varphi\right)_{y}-\left(\partial_{v} \varphi\right)_{x}\right), \\
V_{4}(\varphi) & =\lambda_{0} \sum_{x} \varphi_{x}^{4}+\sum_{Y} \widetilde{V}_{4, Y}, \\
V_{\geqq 6}(\varphi) & =\sum_{Y} V_{\geqq 6, Y},
\end{aligned}
$$

where the various pieces satisfy the conditions for some $C_{ \pm}, \alpha$ :

(a) $C_{-} / n_{0}<\lambda_{0}<C_{+} / n_{0}$,

(b) $m_{0}^{2} \in\left(-n_{0}^{-3 / 2}, n_{0}^{-3 / 2}\right)$,

(c) $\sum_{x \in \Delta, y \in \Delta^{\prime}}\left|K_{\mu \nu}(x, y)\right||x-y|^{2 / 3} \leqq n_{0}^{-3 / 2} \exp \left(-\alpha \mathscr{L}\left(\Delta \cup \Delta^{\prime}\right)\right)$, 
(d) $\widetilde{V}_{4, Y}(\varphi)$ is the restriction to equal arguments of a quartic form $\widetilde{V}_{4, Y}\left(\varphi_{1}, \ldots, \varphi_{4}\right)$ which depends on $\varphi_{4}$ only through $\left(\varphi_{4, x}-\varphi_{4, y}\right)$ and which satisfies for $\varphi_{1}, \ldots, \varphi_{3} \in \mathscr{K}(Y)$ and $\varphi_{4}$ satisfying the bounds of $\mathscr{K}(Y)$ except possibly the first:

$$
\left|\tilde{V}_{4, Y}\right| \leqq n_{0}^{-3 / 4} e^{-\alpha \mathscr{L}(Y)}
$$

(e) $V_{\geqq 6, Y}$ is analytic on $3 \mathscr{K}(Y)$ and satisfies there

$$
\left|V_{\geqq 6, Y}\right| \leqq n_{0}^{-2 / 3} e^{-\alpha \mathscr{L}(Y)} .
$$

The large field region is characterized as follows. For $\varphi \in \mathbb{R}^{\Lambda}$ let $D(\varphi)$ the smallest paved set $D$ such that $|\varphi(x)| \leqq 2 C_{1} n_{0}^{1 / 4} \cdot \exp (1 / 10 \alpha d(x, \sim D))$. Then for any paved set $D$ we define

$$
\mathscr{D}(D, X)=\left\{\varphi \in \mathbb{C}^{X}: \varphi=\varphi_{0}+\varphi_{1}, \varphi_{0} \in \mathbb{R}^{X}, D\left(\varphi_{0}\right) \subset D, \varphi_{1} \in \mathscr{K}(X)\right\} .
$$

The set $\mathscr{D}(D, X)$ includes fields which may be large on $D$, but they must be small off $D$, in fact $\left.\mathscr{D}(D, X)\right|_{X \backslash D} \subset 3 \mathscr{K}(X \backslash D)$. The condition for the large field region is:

GKII. $\left(e^{-V}\right)$ is analytic on $\mathscr{D}(D, X)$. ( $V$ itself need not be defined.) For $\left(\exp \left(-V_{\geqq 4}\right)\right)(\varphi) \equiv e^{V_{2}(\varphi)}\left(e^{-V}\right)(\varphi)$ we have

$$
\left(\exp \left(-V_{\geqq 4}\right)\right)(\varphi)=\sum_{\left\{X_{\omega}\right\}} \prod_{\omega} g_{X_{\omega}}^{D} \exp \left(-\lambda_{0} \sum_{x \in \sim D} \varphi_{x}^{4}-\sum_{Y \subset \sim\left(\bigcup_{\omega} X_{\omega}\right)}\left(\tilde{V}_{4, Y}+V_{\geqq 6, Y}\right)\right),
$$

where the sum is over collections of disjoint paved sets $\left\{X_{\omega}\right\}$ such that $D \subset\left(\bigcup_{\omega} X_{\omega}\right)$ and such that $D \cap X_{\omega}$ is a non-empty union of connected components of $D$. The function $g_{X}^{D}$ are analytic on $\mathscr{D}(D, X)$ and satisfy there

$$
\left|g_{X}^{D}\right| \leqq \exp \left(C_{2}|D \cap X|+\sum_{x \in D \cap X}\left(-\lambda_{0}^{1 / 2}\left|\varphi_{x}\right|^{2}+20 \lambda_{0}\left(\operatorname{Im} \varphi_{x}\right)^{4}\right)-\alpha \mathscr{L}(X)\right) .
$$

There is also an identity the $g_{X}^{D}$ must satisfy ([12], Eq. 4.16).

An example of a model satisfying GKI, GKII is the potential $V(\varphi)=$ $\sum_{x}\left(\frac{1}{2} m_{0}^{2} \varphi_{x}^{2}+\lambda_{0}: \varphi_{x}^{4}:\right)+$ const considered by Gawedzki and Kupiainen. Here $K_{\mu \nu}=0, \widetilde{V}_{4}=0, V_{\geqq 6}=0$, and $g_{X}^{D}=\exp \left(-\lambda_{0} \sum_{x \in X} \varphi_{x}^{4}\right)$ if $X$ is a connected component of $D$ and is zero otherwise.

Given a Hamiltonian $\mathscr{H}_{0}=\frac{1}{2}\left(\varphi, G_{0}^{-1} \varphi\right)+V$ of the above form one defines an effective Hamiltonian $\mathscr{H}_{1}$ on $\Lambda_{N-1}$ by making a block spin transformation:

$$
\exp \left(-\mathscr{H}_{1}\left(\varphi_{1}\right)+\text { const }\right)=\int \exp \left(-\mathscr{H}_{0}(\varphi)\right) \delta\left(\varphi_{1}-C \varphi\right) d \varphi .
$$

Here $C: \mathbb{R}^{\Lambda_{N}} \rightarrow \mathbb{R}^{\Lambda_{N-1}}$ is an operator which averages over blocks of spins with $L^{4}$ points, and then rescales to the unit lattice. The constant is chosen so $\mathscr{H}_{1}(0)=0$. By adjusting an overall constant in $C$ we can arrange that $\mathscr{H}_{1}\left(\varphi_{1}\right)=$ $\frac{1}{2}\left(\varphi_{1}, G_{1}^{-1} \varphi_{1}\right)+V_{1}\left(\varphi_{1}\right)$, where $G_{1}^{-1}$ is a version of the Laplacian on $\mathbb{R}^{\Lambda_{N-1}}$. Iterating this procedure one obtains a sequence of Hamiltonians $\mathscr{H}_{0}, \mathscr{H}_{1}, \mathscr{H}_{2}, \ldots$ 
defined in successively smaller volumes which have the form $\mathscr{H}_{n}\left(\varphi_{n}\right)=$ $\frac{1}{2}\left(\varphi_{n}, G_{n}^{-1} \varphi_{n}\right)+V_{n}\left(\varphi_{n}\right)$.

The main result of Gawedzki-Kupiainen is the following. Let $L$ be sufficiently large, $L \geqq \underline{L}$, and choose constants $\alpha=\alpha(L), C_{ \pm}=C_{ \pm}(L)$. Furthermore let $N_{0}, C_{0}, C_{2}, C_{1}, n_{0}$ be sufficiently large depending on $L$, and chosen in the indicated order so that $N_{0} \geqq \underline{N}_{0}(L), C_{0} \geqq \underline{C}_{0}\left(L, N_{0}\right)$, etc. Suppose that $\mathscr{H}_{0}$ satisfies GKI, GKII so that all couplings are weak. Then for any $\lambda_{0}$ satisfying GKIa one can choose $m_{0}^{2}=f\left(\lambda_{0}\right)$ satisfying GKIb so that $V_{n}$ satisfies GKI, GKII with $n_{0}$ replaced by $n_{0}+n$ for all $n$. (Actually one must express $V_{n}$ in a new variable $\psi_{n}$, a smeared version of $\varphi_{n}$, for this to be true.)

The small field region eventually includes everything, and so in a certain sense we have $\lim _{n \rightarrow \infty} V_{n}=0$. That is, all variables in the potential are irrelevant (once we adjust the mass). One can also establish a sense in which $\lim _{n \rightarrow \infty} G_{n}^{-1}=G_{*}^{-1}$, a version of the continuum Laplacian. Thus the flow of the renormalization group is toward a free massless boson field theory. This is the infrared asymptotic freedom.

The renormalization group results give one control over the model. One can prove that the infinite volume limit exists for the correlation functions. Furthermore correlations only decay as $|x-y|^{-2}$ as $|x-y| \rightarrow \infty$, confirming that we have a critical theory. For these results and more see [13].

\section{The Pseudoscalar Yukawa Model}

The Hamiltonian $\mathscr{H}_{0}$ for the pseudoscalar Yukawa model depends on a boson field $\varphi$ as above and on fermion fields $\psi, \tau$ which are elements of a Grassman algebra. It has the form

$$
\begin{aligned}
\mathscr{H}_{0}(\varphi, \tilde{\psi}, \psi)= & \frac{1}{2} \sum_{x, v} \varphi_{x}\left(G_{0}^{-1}\right)_{x y} \varphi_{y}+\sum_{x}\left(\frac{1}{2} m_{0}^{2} \varphi_{x}^{2}+\lambda_{0}: \varphi_{x}^{4}:\right) \\
& +\sum_{x, y} \widetilde{\psi}_{x}\left(S_{0}^{-1}\right)_{x y} \psi_{y}+g \sum_{x} \widetilde{\psi}_{x}\left(i \gamma_{5}\right) \psi_{x} \varphi_{x},
\end{aligned}
$$

where $S_{0}^{-1}$ is a lattice version of the Dirac operator with mass $M$. The presence of the $\varphi^{4}$ term is standard for this model (see for example [16].).

We immediately integrate out the fermions to obtain an effective boson theory. This amounts to restricting attention to the charge zero sector. The new Hamiltonian $\mathscr{H}_{0}(\varphi)$ is defined by

$$
e^{\left(-\mathscr{H}_{0}(\varphi)+\text { const }\right)}=\int e^{-\mathscr{H}_{0}(\varphi, \tilde{\psi}, \psi)} d \tilde{\psi} d \psi
$$

with the constant adjusted so $\mathscr{H}_{0}(0)=0$. We obtain

$$
\mathscr{H}_{0}(\varphi)=\frac{1}{2} \sum_{x, y} \varphi_{x}\left(G_{0}^{-1}\right)_{x y} \varphi_{y}+\sum_{x}\left(\frac{1}{2} m_{0}^{2}-6 \lambda_{0} G_{0, x x}\right) \varphi_{x}^{2}+\hat{\lambda}_{0} \sum_{x} \varphi_{x}^{4}-\log \Omega(\varphi)
$$

where

$$
\Omega(\varphi)=\operatorname{det}\left(\mathbb{1}+g S_{0}\left(i \gamma_{5}\right) \varphi\right) .
$$


Our goal is to show that $\mathscr{H}_{0}$ (or $e^{-\mathscr{H}_{0}}$ ) satisfies GKI, GKII. This involves extracting corrections to $m_{0}^{2}, \lambda_{0}$ from $\log \Omega$ and showing that the rest is irrelevant. The details are similar to the analysis of [12].

We begin by defining $S_{0}$ more precisely. Let $M>0$ be a fixed constant and let $\gamma_{\mu}$ be four dimensional Dirac matrices satisfying $\gamma_{\mu} \gamma_{\nu}+\gamma_{\nu} \gamma_{\mu}=-2 \delta_{\mu \nu}, \gamma_{\mu}^{*}=-\gamma_{\mu}$. Then $S_{0}$ is the operator on $\left(\mathbb{C}^{4}\right)^{A}$ given by the matrix

$$
\left(S_{0}\right)_{\alpha \beta}(x, y)=|\Lambda|^{-1} \sum_{p \in X} e^{i p(x-y)}\left(\sum_{\mu} \gamma_{\mu} \sin p_{\mu}+M\right)_{\alpha \beta}^{-1},
$$

where $\tilde{\Lambda}$ is the toroidal lattice:

$$
\tilde{\Lambda}=\tilde{\Lambda}_{N}=\left(2 \pi L^{-N} \mathbb{Z} / 2 \pi \mathbb{Z}\right)^{4} \cong\left([-\pi, \pi] \cap 2 \pi L^{-N} \mathbb{Z}\right)^{4} .
$$

The factor $\widetilde{S}_{0}(p)=\left(\sum_{\mu} \gamma_{\mu} \sin p_{\mu}+M\right)^{-1}$ is analytic and bounded on a strip containing the real axis, from which we deduce that for some $\mu>0$ and sufficiently small (depending on $M$ )

$$
\left|\left(S_{0}\right)_{\alpha \beta}(x, y)\right| \leqq C e^{-\mu|x-y|} .
$$

This estimates and all subsequent estimates are uniform in the volume (i.e. uniform in $N$ ). The estimate is best proved first in infinite volume by deforming the contour and then in finite volume by periodizing. Some variation in the form of $S_{0}$ is tolerable. In particular if we wanted to study the continuum limit we should take $\tilde{S}_{0}(p)=\left(\sum_{\mu} \gamma_{\mu} \sin p_{\mu}+\sum_{\mu}\left(1-\cos p_{\mu}\right)+M\right)^{-1}$. (cf. [18]).

Next we show that $\Omega(\varphi)$ has the general form required of our potentials.

Lemma 1. (a) $\Omega$ is real,

(b) $\Omega(\varphi)=\Omega(-\varphi)$,

(c) $\Omega$ has all the symmetries of the lattice.

Note. The identity $\Omega(\varphi)=\Omega(-\varphi)$, which is Furry's Theorem, is not true for the scalar Yukawa model. Our proof for the pseudoscalar model follows Seiler [17] who considers the two-dimensional case. The result seems to be well-known in the older literature, but I have been unable to find a reference.

Proof. (a) Choose a representation where $\gamma_{0}$ is real and $\gamma_{1}, \gamma_{2}, \gamma_{3}$ are imaginary. (For example, consider the Majoranna representation of the Lorentzian Dirac matrices in which all $\gamma_{\mu}$ are imaginary. Then replace $\gamma_{0}$ by $i \gamma_{0}$ to obtain a Euclidean representation of required type.) Then $\gamma_{5} \equiv \gamma_{0} \gamma_{1} \gamma_{2} \gamma_{3}$ is imaginary and we have $\gamma_{0} \gamma_{\mu} \gamma_{0}=-\bar{\gamma}_{\mu}$ and hence $\bar{S}_{0}=\gamma_{0} S_{0} \gamma_{0}$. Thus

$$
\overline{\Omega(\varphi)}=\operatorname{det}\left(1+g \bar{S}_{0}\left(\overline{i \gamma_{5}}\right) \varphi\right)=\operatorname{det}\left(1+g \gamma_{0} S_{0} \gamma_{0}\left(i \gamma_{5}\right) \varphi\right)=\Omega(\varphi),
$$

since $\gamma_{0} \gamma_{5}=-\gamma_{5} \gamma_{0}$ and $\gamma_{0}^{2}=-1$. Thus $\Omega$ is real.

(b) On the other hand since $S_{0}^{*}=\gamma_{5} S_{0} \gamma_{5}, \gamma_{5}^{*}=\gamma_{5}$, and $\gamma_{5}^{2}=1$,

$$
\begin{aligned}
\bar{\Omega} \overline{(\varphi)} & =\operatorname{det}\left(1+g\left(S_{0}\left(i \gamma_{5}\right) \varphi\right)^{*}\right)=\operatorname{det}\left(1-g \varphi\left(i \gamma_{5}\right) \gamma_{5} S_{0} \gamma_{5}\right) \\
& =\operatorname{det}\left(1-g \varphi S_{0}\left(i \gamma_{5}\right)\right)=\Omega(-\varphi) .
\end{aligned}
$$


(c) $\Omega$ is invariant under lattice symmetries since the original interaction is invariant. In particular if $\pi_{\mu}$ is the reflection thru the plane $x_{\mu}=0$ then $\Omega(\varphi)=$ $\Omega\left(-\varphi \circ \pi_{\mu}\right)$ by the structure of $\Omega$ and hence $\Omega(\varphi)=\Omega\left(\varphi \circ \pi_{\mu}\right)$ by (b).

Next we note the identity (cf. [19])

$$
\Omega=\exp \left(-\sum_{k=1}^{n-1} W_{k}\right) \Omega_{\geqq n}
$$

where

$$
W_{k}=(-1)^{k} k^{-1} \operatorname{Tr}\left(\left(g S_{0} i \gamma_{5} \varphi\right)^{k}\right), \quad \Omega_{\geqq n}=\operatorname{det}_{n}\left(1+g S_{0} i \gamma_{5} \varphi\right) .
$$

This gives the expansion of $\log \Omega$ in powers of $\varphi$ or $g$, i.e. perturbation theory. We have $\log \Omega=-\sum_{k=1}^{n-1} W_{k}+\log \Omega_{\geqq n}$ (at least for $\varphi$ or $g$ small). By the lemma $W_{k}=0$ for $k$ odd. Then $\Omega=\Omega_{\geqq 2}$ and so we have the basic estimate for all $g \in \mathbb{R}, \varphi \in \mathbb{C}^{A}$

$$
\begin{aligned}
|\Omega| & \leqq \exp \left(\operatorname{Tr}\left(\left(g S_{0} i \gamma_{5} \varphi\right)^{*}\left(g S_{0} i \gamma_{5} \varphi\right)\right)\right) \\
& =\exp \left(g^{2} \sum_{x, y}|\varphi(x)|^{2} \operatorname{Tr}\left(S_{0}(x, y) \gamma_{5} S_{0}(y, x) \gamma_{5}\right)\right) \\
& \leqq \exp \left(\mathcal{O}\left(g^{2}\right) \sum_{x}|\varphi(x)|^{2}\right) .
\end{aligned}
$$

We study $W_{2}, W_{4}$ in more detail. We have

$$
W_{2}=\frac{1}{2} g^{2} \sum_{x, y, z} \varphi(x) \operatorname{Tr}\left(S_{0}(x, z)\left(i \gamma_{5}\right) S_{0}(z, y)\left(i \gamma_{5}\right)\right) \varphi(y) \equiv \sum_{x, y} \varphi(x) K(x, y) \varphi(y),
$$

and

$$
\begin{aligned}
W_{4} & =\frac{1}{4} g^{4} \sum_{x_{1}, \ldots, x_{4}} \operatorname{Tr}\left(S_{0}\left(x_{1}, x_{2}\right)\left(i \gamma_{5}\right) \cdots S\left(x_{4}, x_{1}\right)\left(i \gamma_{5}\right)\right) \varphi\left(x_{1}\right) \cdots \varphi\left(x_{4}\right) \\
& \equiv \sum_{x_{1}, \ldots, x_{4}} \Lambda\left(x_{1}, \ldots, x_{4}\right) \varphi\left(x_{1}\right) \cdots \varphi\left(x_{4}\right) .
\end{aligned}
$$

\section{Lemma 2}

(a) $W_{2}=\frac{1}{2} \zeta_{0} \sum_{x, \mu}\left(\partial_{\mu} \varphi\right)_{x}^{2}+\frac{1}{2} \delta m_{0}^{2} \sum_{x} \varphi_{x}^{2}+\sum_{x, y \mu, \nu} \sum_{\mu}\left(\partial_{\mu} \varphi\right)_{x} K_{\mu \nu}(x, y)\left(\left(\partial_{v} \varphi\right)_{y}-\left(\partial_{v} \varphi\right)_{x}\right)$, where $\zeta_{0}, \delta m_{0}^{2}$ are $\mathcal{O}\left(g^{2}\right)$ and

$$
\left|K_{\mu \nu}(x, y)\right| \leqq \mathcal{O}\left(g^{2}\right) e^{-\mu|x-y|} .
$$

(b) $W_{2}=\sum_{Y} W_{2, Y}$, where the sum is over paved subsets of $\Lambda$, with $1 \leqq|Y| \leqq 2, W_{2, Y}$ depends on $\left\{\varphi_{x}\right\}_{x \in Y}$ and

$$
\left|W_{2, Y}\right| \leqq \mathcal{O}\left(g^{2}\right) e^{-\mu \mathscr{L}(Y)} \sum_{x \in Y}\left|\varphi_{x}\right|^{2} .
$$


Note. Here and subsequently an expression like $\mathcal{O}\left(g^{2}\right)$ means bounded by $C g^{2}$, where $C$ may depend on $L, N_{0}$ the parameters of the paving.

Proof. (a) Since $K(x, y)=K(x-y)$ and $K(x)=\mathcal{O}\left(g^{2} e^{-\mu|x|}\right)$ for some $\mu>0$ we have that $\tilde{K}(p)=\sum_{x} e^{-i p x} K(x)$ is bounded by $\mathcal{O}\left(g^{2}\right)$ and analytic in a strip $\left|\operatorname{Im} p_{\mu}\right|<\mu$ for some $\mu>0$. Since $K(x)=K(-x)$ we have $\left(\partial_{\mu} \tilde{K}\right)(0)=0$. It follows that ([12], Appendix 2)

$$
\tilde{K}(p)=\tilde{K}(0)+\sum_{\mu, \nu} \sum_{p}\left(e^{i p_{\mu}}-1\right)\left(e^{i p_{\nu}}-1\right) A_{\mu \nu}(p),
$$

where $A_{\mu \nu}(p)$ is $\mathcal{O}\left(g^{2}\right)$ and analytic in any smaller strip $\left|\operatorname{Im} p_{\mu}\right|<\mu$. Correspondingly we have

$$
W_{2}=\tilde{K}(0) \sum_{x} \varphi_{x}^{2}+\sum_{x, y} \sum_{\mu, v}\left(\partial_{\mu} \varphi\right)_{x} K_{\mu \nu}(x, y)\left(\partial_{v} \varphi\right)_{y},
$$

where $K_{\mu v}(x, y)=K_{\mu \nu}(x-y)$ satisfies the bound of the lemma. One can arrange that $K_{\mu \nu}$ has all the symmetries of the lattice.

Now replace $\left(\partial_{v} \varphi\right)_{y}$ by $\left(\left(\partial_{v} \varphi\right)_{y}-\left(\partial_{v} \varphi\right)_{x}\right)+\left(\partial_{v} \varphi\right)_{x}$. In the second term we get $\sum_{y} K_{\mu v}(x-y)=\widetilde{K}_{\mu \nu}(0)$. By the lattice symmetries this is proportional to $\delta_{\mu \nu}$. Thus the lemma follows with

$$
\zeta_{0} \delta_{\mu \nu}=2 \widetilde{K}_{\mu \nu}(0), \quad \delta m_{0}^{2}=2 \widetilde{K}(0) .
$$

(b) The representation holds with

$$
\begin{aligned}
W_{2, Y} & =\sum_{x, y} K_{Y}(x, y) \varphi_{x} \varphi_{y}, \\
K_{Y}(x, y) & =\sum_{\Delta_{1} \cup \Delta_{2}=Y} \chi_{\Delta_{1}}(x) K(x, y) \chi_{\Delta_{2}}(y) .
\end{aligned}
$$

Note that $K_{Y}$ has support in $Y \times Y$. We estimate this by

$$
\left|W_{2, Y}\right| \leqq\left\|K_{Y}\right\|_{2}\|\varphi\|_{2, Y}^{2},
$$

where $\|\varphi\|_{2, Y}^{2}=\sum_{x \in Y}\left|\varphi_{x}\right|^{2}$. Now from the estimate on $K$

$$
\left|\chi_{\Delta_{1}}(x) K(x, y) \chi_{\Delta_{2}}(y)\right| \leqq \mathcal{O}\left(g^{2}\right) e^{-\mu d\left(\Delta_{1}, \Delta_{2}\right)} .
$$

But

$$
d\left(\Delta_{1}, \Delta_{2}\right) \geqq \mathscr{L}\left(\Delta_{1} \cup \Delta_{2}\right)-2 L^{N_{0}},
$$

and so

$$
\left|K_{Y}\right| \leqq \mathcal{O}\left(g^{2}\right) e^{-\mu \mathscr{L}(Y)} .
$$

This gives the same estimate on $\left\|K_{Y}\right\|_{2}$ since $\operatorname{Vol} \Delta=L^{4 N_{0}}=\mathcal{O}(1)$. 


\section{Lemma 3.}

(a) $W_{4}=\sum_{Y} W_{4, Y}$, where $1 \leqq|Y| \leqq 4$ and

$$
\left|W_{4, Y}\right| \leqq \mathcal{O}\left(g^{4}\right) e^{-\mu \mathscr{L}(Y)} \sum_{x \in Y}\left|\varphi_{x}\right|^{4}
$$

(b) $W_{4}=\delta \lambda_{0} \sum_{x} \varphi_{x}^{4}+\tilde{W}_{4}$, where $\delta \lambda_{0}=\mathcal{O}\left(g^{4}\right)$.

Furthermore $\tilde{W}_{4}=\sum_{Y} \tilde{W}_{4, Y}$, where $\tilde{W}_{4, Y}$ satisfies the same bound as $W_{4, Y}$ in (a)

Proof.

(a) For $1 \leqq|Y| \leqq 4$ we define $W_{4, Y}$ with kernel $\Lambda_{Y}$ defined by

$$
\Lambda_{Y}\left(x_{1}, \ldots, x_{4}\right)=\sum_{\Delta_{1} \cup \ldots \cup \Delta_{4}=Y} \chi_{\Delta_{1}}\left(x_{1}\right) \cdots \chi_{\Delta_{4}}\left(x_{4}\right) \Lambda\left(x_{1}, \ldots, x_{4}\right) .
$$

Then by Hölder's inequality

$$
\left|W_{4, Y}\right| \leqq\left\|\Lambda_{Y}\right\|_{4 / 3}\|\varphi\|_{4, Y}^{4} .
$$

But we have

$$
\left|\Lambda_{Y}\right| \leqq \mathcal{O}\left(g^{4}\right) e^{-\mu \mathscr{L}(Y)}
$$

which gives the same bound for $\left\|\Lambda_{Y}\right\|_{4 / 3}$ and hence the result.

(b) We insert $\varphi_{x_{2}}=\left(\varphi_{x_{2}}-\varphi_{x_{1}}\right)+\varphi_{x_{1}}$ in $W_{4}$, then in the term with $\varphi_{x_{1}}^{2}$ we insert $\varphi_{x_{3}}=\left(\varphi_{x_{3}}-\varphi_{x_{1}}\right)+\varphi_{x_{1}}$, etc. The $\varphi_{x_{1}}$ terms are collected to give $\delta \lambda_{0} \sum_{x} \varphi_{x}^{4}$ with

$$
\delta \lambda_{0}=\sum_{x_{2}, x_{3}, x_{4}} \Lambda\left(0, x_{2}, x_{3}, x_{4}\right)
$$

which is $\mathcal{O}\left(g^{4}\right)$. The remaining terms are collected to give $\tilde{W}_{4}$. We have $\tilde{W}_{4}=$ $\sum_{Y} \tilde{W}_{4, Y}$ with

$$
\tilde{W}_{4, Y}=\sum_{i=2}^{4} \sum_{x_{1}, \ldots, x_{4}} \Lambda_{Y}\left(x_{1}, \ldots, x_{4}\right) \varphi_{x_{1}}^{i-1}\left(\varphi_{x_{2}}-\varphi_{x_{1}}\right) \varphi_{x_{i+1}} \cdots \varphi_{x_{4}}
$$

Using the bound on $\Lambda_{Y}$ above each monomial in this sum can be estimated by the required $\mathcal{O}\left(g^{4}\right) e^{-\mu \mathscr{L}(Y)} \sum_{x \in Y}\left|\varphi_{x}\right|^{4}$.

Remark. The expression for $\tilde{W}_{4, Y}(\varphi)$ is the restriction to equal arguments of

$$
\begin{aligned}
& \tilde{W}_{4, Y}\left(\varphi_{1}, \ldots, \varphi_{4}\right) \\
& \quad=\sum_{i=2}^{4} \sum_{x_{1}, \ldots, x_{4}} \Lambda_{Y}\left(x_{1}, \ldots, x_{4}\right) \varphi_{1, x_{1}} \cdots \varphi_{i-1, x_{1}} \varphi_{i, x_{i+1}} \cdots \varphi_{3, x_{4}}\left(\varphi_{4, x_{1}}-\varphi_{4, x_{1}}\right)
\end{aligned}
$$

which depends on $\varphi_{4}$ only through difference variables. 


\section{Expansions for the Fermion Determinant $\boldsymbol{\Omega}$}

We now want to analyze the locality properties of $\log \Omega$. This involves making a number of expansions and estimating the terms in the expansion. For all the estimates in this section the bounds are asserted to hold for $\mu>0$ and sufficiently small, $L \geqq L(\mu), N_{0} \geqq N_{0}(\mu, L)$.

We begin by making a cluster expansion. $([5,13,15,18])$

\section{Lemma 4.}

$$
\Omega=\sum_{\left\{X_{i}\right\}} \prod_{i} \rho_{X_{i}}
$$

where the sum is over all partitions $\left\{X_{i}\right\}$ of $\Lambda$ and $\rho_{X_{1}}$ depends only on $\varphi \uparrow X_{i}$. For all $\varphi \in \mathbb{C}^{\Lambda}, g \in \mathbb{R},($ and say $|g| \leqq 1$ )

$$
\begin{gathered}
\left|\rho_{\Delta}\right| \leqq \exp \left(\mathcal{O}\left(g^{2}\right) \sum_{x \in \Delta}\left|\varphi_{x}\right|^{2}\right) \\
\left|\rho_{X}\right| \leqq \mathcal{O}\left(|g|^{1 / 2}\right) e^{-\mu \mathscr{L}(X)} \exp \left(\mathcal{O}(|g|) \sum_{x \in X}\left|\varphi_{x}\right|^{2}\right), \quad|X| \geqq 2 .
\end{gathered}
$$

Proof. For each pair of distinct blocks $\Delta, \Delta^{\prime}$ introduce a variable $0 \leqq s_{\Delta, \Delta^{\prime}} \leqq 1$, let $s=\left\{s_{\Delta, \Delta^{\prime}}\right\}$, and define

$$
S_{0}^{s}(x, y)=\sum_{\Delta} \chi_{\Delta}(x) S_{0}(x-y) \chi_{\Delta}(y)+\sum_{\Delta \neq \Delta^{\prime}} \chi_{\Delta}(x) S_{0}(x-y) \chi_{\Delta}(y) s_{\Delta, \Delta^{\prime}} .
$$

Then

$$
\Omega=\sum_{\Gamma} \int d s_{\Gamma} \partial / \partial s_{\Gamma}\left[\operatorname{det}\left(1+g S_{0}^{s}\left(i \gamma_{5}\right) \varphi\right)\right]_{s_{\sim T}=0}
$$

where the sum is over collections $\Gamma$ of pairs $\left(\Delta, \Delta^{\prime}\right)$ and $s_{\Gamma}=\left\{s_{\Delta, \Delta^{\prime}}\right\}_{\left(\Delta, \Delta^{\prime}\right) \in \Gamma}$. Now $\Gamma$ determines a partition $\left\{X_{i}\right\}$ of $\Lambda$ by grouping together the blocks it connects. Since $S_{0}^{s}$ with $s_{\sim \Gamma}=0$ preserves each subspace in $\left(\mathbb{C}^{4}\right)^{\Lambda}=\oplus_{i}\left(\mathbb{C}^{4}\right)^{X_{i}}$ the determinant factors. Summing first over $\left\{X_{i}\right\}$ and then over compatible $\Gamma$ 's we have the claimed representation for $\Omega$ with

$$
\rho_{X}=\sum_{\Gamma \operatorname{conn} X} \int d s_{\Gamma} \partial /\left.\partial s_{\Gamma} \operatorname{det}_{X}\left(1+g S_{0}^{s_{T}}\left(i \gamma_{5}\right) \varphi\right)\right|_{s_{X} \sim \Gamma=0},
$$

where the sum is over all $\Gamma^{\prime}$ 's connecting $X$ and $\operatorname{det}_{X}(\cdot)$ means the determinant of the operator on $\left(\mathbb{C}^{4}\right)^{X}$.

To estimate the derivatives $\partial / \partial s_{\Gamma}$ we allow $s_{\Gamma}$ to be complex. We take $s_{\Gamma}$ in the polydisc

$$
\left|s_{\Delta, \Delta^{\prime}}\right| \leqq 2 r e^{\mu d\left(\Delta, \Delta^{\prime}\right)}, \quad\left(\Delta, \Delta^{\prime}\right) \in \Gamma,
$$

where $\mu$ is small enough so $\left|S_{0}(x, y)\right| \leqq C e^{-2 \mu|x-y|}$ and $r \geqq 1$. In this domain $\left|S_{0}^{s_{\Gamma}}(x, y)\right| \leqq C r e^{-\mu|x-y|}$ and it follows as in the estimate on $\Omega$ that

$$
\left|\operatorname{det}_{X}\left(\mathbb{1}+g S^{s}\left(i \gamma_{5}\right) \varphi\right)\right| \leqq \exp \left(\mathcal{O}\left(g^{2} r^{2}\right) \sum_{x \in X}\left|\varphi_{x}\right|^{2}\right) \text {. }
$$


Also in this domain we can surround any point with $\left|s_{\Delta, \Delta^{\prime}}\right| \leqq 1$ by a polydisc whose radius in $s_{\Delta, \Delta^{\prime}}$ is $r e^{\mu d\left(\Delta, \Delta^{\prime}\right)}$. Thus for $\left|s_{\Delta, \Delta^{\prime}}\right| \leqq 1$ we have by the Cauchy bounds:

$$
\begin{aligned}
& \left|\partial / \partial s_{\Gamma} \operatorname{det}_{X}\left(1+g S^{s}\left(i \gamma_{5}\right) \varphi\right)\right| \\
& \quad \leqq\left[\prod_{\left(\Delta, \Delta^{\prime}\right) \in \Gamma} r^{-1} e^{-\mu d\left(\Delta, \Delta^{\prime}\right)}\right] \exp \left(\mathcal{O}\left(g^{2} r^{2}\right) \sum_{x \in X}\left|\varphi_{x}\right|^{2}\right) .
\end{aligned}
$$

If $|X| \geqq 2$, then $\Gamma$ is not empty and we can extract a factor $r^{-1}$ from this expression. Using also $d\left(\Delta_{1}, \Delta_{2}\right) \geqq \mathscr{L}\left(\Delta_{1} \cup \Delta_{2}\right)-2 L^{N_{0}}$ the expression in brackets is bounded by $\mathcal{O}\left(r^{-1}\right) \Pi_{\left(\Delta, \Delta^{\prime}\right) \in \Gamma} \exp \left(-\mu \mathscr{L}\left(\Delta \cup \Delta^{\prime}\right)\right)$.

Each $\Gamma$ can be thought of as a graph on the blocks $\Delta$ in $X$. By deleting lines in $\Gamma$ we can break all closed loops and obtain a tree graph which still connects $X$. This leads to the bound

$$
\Pi_{\left(\Delta, \Delta^{\prime}\right) \in \Gamma} \exp \left(-(\mu / 2) \mathscr{L}\left(\Delta \cup \Delta^{\prime}\right)\right) \leqq \exp (-(\mu / 2) \mathscr{L}(X)) .
$$

On the other hand the sum over $\Gamma$ can be estimated by

$$
\sum_{\Gamma\left(\Delta, \Delta^{\prime}\right) \in \Gamma} e^{-\frac{1}{2} \mathscr{L}\left(\Delta \cup \Delta^{\prime}\right)} \leqq \sum_{\left\{Y_{\alpha}\right\}} \prod_{\alpha} e^{-\frac{1}{2} \mu \mathscr{L}\left(Y_{\alpha}\right)} \leqq e^{2|X|} .
$$

Here the second sum is over all collections $\left\{Y_{\alpha}\right\}$ of paved subsets with $Y_{\alpha} \subset X$ and $\left|Y_{\alpha}\right|=2$. The final estimate, valid without the restriction $\left|Y_{\alpha}\right|=2$, follows from the general bound $\sum_{Y \subset X} \exp (-\gamma \mathscr{L}(Y)) \leqq 2|X|$ valid for $N_{0} \geqq \underline{N}_{0}(\gamma)$ sufficiently large.

Combine the above estimates using $\mathscr{L}(X) \geqq L^{N_{0}}(|X|-1)$, hence $\mathscr{L}(X) \geqq \frac{1}{2} L^{N_{0}}|X|$ for $|X| \geqq 2$, and hence $2|X|-(\mu / 4) \mathscr{L}(X)<0$ for $N_{0}$ sufficiently large. Then we have

$$
\left|\rho_{X}\right| \leqq \mathcal{O}\left(r^{-1}\right) e^{-(\mu / 4) \mathscr{L}(X)} \exp \left(\mathcal{O}\left(g^{2} r^{2}\right) \sum_{x \in X}\left|\varphi_{x}\right|^{2}\right) .
$$

Taking $r=|g|^{-1 / 2}$ gives the result.

If $|X|=1$ then $X=\Delta$ and

$$
\rho_{\Delta}=\operatorname{det}_{\Delta}\left(1+g S_{0, \Delta}\left(i \gamma_{5}\right) \varphi\right)
$$

where $S_{0, \Delta}(x, y)=\chi_{\Delta}(x) S_{0}(x, y) \chi_{\Delta}(y)$. We have as before $\left|\rho_{\Delta}\right| \leqq \exp \left(\mathcal{O}\left(|g|^{2}\right) \sum_{x \in \Delta}\left|\varphi_{x}\right|^{2}\right)$. have

We also need an expansion for $\Omega_{\geqq 6}$. Under the same hypotheses as Lemma 4 we

Lemma 5. $\Omega_{\geqq 6}=\sum_{\left\{X_{\gamma}\right\}} \prod_{\gamma} \rho_{X_{\gamma}}^{\geqq 6}$,

where

$$
\begin{aligned}
& \left|\rho_{\Delta}^{\geqq 6}\right| \leqq \exp \left(\mathcal{O}\left(g^{2}\right) \sum_{x \in \Delta}\left(\left|\varphi_{x}\right|^{2}+\left|\varphi_{x}\right|^{4}\right)\right), \\
& \left|\rho_{X}^{\geqq 6}\right| \leqq \mathcal{O}\left(|g|^{1 / 2}\right) e^{-\mu \mathscr{L}(X)} \exp \left(\mathcal{O}(|g|) \sum_{x \in X}\left(\left|\varphi_{x}\right|^{2}+\left|\varphi_{x}\right|^{4}\right)\right), \quad|X| \geqq 2 .
\end{aligned}
$$

Proof. We have $\Omega_{\geqq 6}=e^{W_{2}+W_{4}} \Omega$. Expand $\Omega$ by the cluster expansion in Lemma 4 , 
and make a Mayer expansion for $e^{W_{2}}$ and $e^{W_{4}}$ using Lemma 2 and Lemma 3. For example

$$
e^{W_{2}}=\exp \left(\sum_{Y} W_{2, Y}\right)=\prod_{Y}\left(\left(e^{W_{2, Y}}-1\right)+1\right)=\sum_{\left\{Y_{\alpha}\right\}} \prod_{\alpha}\left(\exp \left(W_{2, Y_{\alpha}}\right)-1\right),
$$

where the sum is over collections $\left\{Y_{\alpha}\right\}$ of distinct subsets $Y_{\alpha}$ of $\Lambda$ with $1 \leqq\left|Y_{\alpha}\right| \leqq 2$. Similarly $e^{W_{4}}$ is expressed as a sum over $\left\{Y_{\beta}\right\}$ with $1 \leqq\left|Y_{\beta}\right| \leqq 4$. Thus we have

$$
\Omega_{\geqq 6}=\sum_{\left\{X_{i}\right\},\left\{Y_{\alpha}\right\},\left\{Y_{\beta}\right\}} \prod_{i} \rho_{X_{i}} \prod_{\alpha}\left(\exp W_{2, Y_{\alpha}}-1\right) \prod_{\beta} \exp \left(W_{4, Y_{\beta}}-1\right) .
$$

Any choice $\left\{X_{i}\right\},\left\{Y_{\alpha}\right\},\left\{Y_{\beta}\right\}$ determines a new partition $\left\{X_{\gamma}\right\}$ which is the finest partition such that each $X_{i}, Y_{\alpha}, Y_{\beta}$ is contained in only one $X_{\gamma}$. If we fix $\left\{X_{\gamma}\right\}$ the sum factors into a product and we have the claimed representation for $\Omega_{\geqq 6}$ with

$$
\rho_{\bar{X}}^{\geqq 6}=\sum_{\left\{X_{i}\right\},\left\{Y_{\alpha}\right\},\left\{Y_{\beta}\right\} \rightarrow X} \prod_{i} \rho_{X_{i}} \prod_{\alpha}\left(\exp W_{2, Y_{\alpha}}-1\right) \prod_{\beta}\left(\exp W_{4, Y_{\beta}}-1\right) .
$$

Here the sum is over all $\left\{X_{i}\right\},\left\{Y_{\alpha}\right\},\left\{Y_{\beta}\right\}$ such that $\left\{X_{i}\right\}$ is a partition of $X$, each $Y_{\alpha}, Y_{\beta} \subset X$, and $X$ is connected with respect to $\left\{X_{i}\right\},\left\{Y_{\alpha}\right\},\left\{Y_{\beta}\right\}$, i.e. $X$ cannot be divided without dividing one of these sets. (However, $X$ is not necessarily connected.)

Since $\left|e^{W}-1\right| \leqq|W| e^{|W|}$ we have

$$
\begin{aligned}
\left|\exp \left(W_{2, Y_{\alpha}}\right)-1\right| & \leqq\left(\mathcal{O}\left(g^{2}\right) e^{-\mu \mathscr{L}\left(Y_{\alpha}\right)} \sum_{x \in Y_{\alpha}}\left|\varphi_{x}\right|^{2}\right) \exp \left(\mathcal{O}\left(g^{2}\right) e^{-\mu \mathscr{L}\left(Y_{\alpha}\right)} \sum_{x \in Y_{\alpha}}\left|\varphi_{x}\right|^{2}\right) \\
& \leqq \mathcal{O}(|g|) e^{-(\mu / 2) \mathscr{L}\left(Y_{\alpha}\right)} \exp \left(\mathcal{O}(|g|) e^{-(\mu / 2) \mathscr{L}\left(Y_{\alpha}\right)} \sum_{x \in Y_{\alpha}}\left|\varphi_{x}\right|^{2}\right) .
\end{aligned}
$$

To combine the exponentials in the product over $\alpha$ we use

$$
\sum_{\alpha} e^{-(\mu / 2) \mathscr{L}\left(Y_{\alpha}\right)} \sum_{x \in Y_{\alpha}}\left|\varphi_{x}\right|^{2}=\sum_{x \in \cup_{\alpha} Y_{\alpha}}\left(\sum_{\alpha: Y_{\alpha} \in X} e^{-(\mu / 2) \mathscr{L}\left(Y_{\alpha}\right)}\right)\left|\varphi_{x}\right|^{2} \leqq 2 \sum_{x \in X}\left|\varphi_{x}\right|^{2} .
$$

(Here we use $\sum_{Y: A \subset Y} e^{-\gamma \mathscr{L}(Y)} \leqq 2$ valid for $N_{0} \geqq \underline{N}_{0}(\gamma)$.) Using a similar estimate for the $W_{4, Y_{\beta}}$ terms and the estimate on $\rho_{X_{i}}$ from Lemma 4 we obtain for $|X| \geqq 2$

$$
\begin{aligned}
\rho_{\bar{X}}^{\geqq} \leqq & {\left[\sum_{\left\{X_{i}\right\},\left\{Y_{\alpha}\right\},\left\{Y_{\beta}\right\} \rightarrow X} \prod_{i} e^{-(\mu / 2) \mathscr{L}\left(X_{i}\right)} \prod_{\alpha} e^{-(\mu / 2) \mathscr{L}\left(Y_{\alpha}\right)} \prod_{\beta} e^{-(\mu / 2) \mathscr{L}\left(Y_{\beta}\right)}\right] } \\
& \cdot \mathcal{O}\left(|g|^{1 / 2}\right) \exp \left(\mathcal{O}(|g|) \sum_{x \in X}\left(\left|\varphi_{x}\right|^{2}+\left|\varphi_{x}\right|^{4}\right)\right) .
\end{aligned}
$$

The factor $\mathcal{O}\left(|g|^{1 / 2}\right)$ may be extracted since each term in the sum either has an $X_{i}$ with $\left|X_{i}\right| \geqq 2$ or a $Y_{\alpha}$ or a $Y_{\beta}$.

Now using

$$
\sum_{i} \mathscr{L}\left(X_{i}\right)+\sum_{\alpha} \mathscr{L}\left(Y_{\alpha}\right)+\sum_{\beta} \mathscr{L}\left(Y_{\alpha}\right) \geqq \mathscr{L}(X)
$$

we extract a factor $e^{-(\mu / 4) \mathscr{L}(X)}$ from the bracketed sum. The remaining sums can be 
estimated by $\left(e^{2|X|}\right)^{3}$ as explained earlier. Then since $6|X|-\mu / 8 \mathscr{L}(X)<0$ for $N_{0}$ sufficiently large, the bracketed sum is bounded by $e^{-(\mu / 8) \mathscr{L}(X)}$. This gives our result.

If $X=\Delta$ then

$$
\rho_{\Delta}^{\geqq 6}=\rho_{\Delta} \exp \left(W_{2, \Delta}+W_{4, \Delta}\right),
$$

and the result follows from the earlier bounds on these objects.

The remaining results in this section consist of refinements that are appropriate when $\varphi$ is either in the small field region $\mathscr{K}(\Lambda)$ or the large field region $\mathscr{D}(D, X)$ as defined previously. These results hold for $g$ sufficiently small depending on everything, i.e. $|g| \leqq \underline{g}\left(\mu, L, N_{0}, C_{0}, C_{2}, C_{1}, n_{0}\right)$.

Lemma 6. For $\varphi \in 6 \mathscr{K}(\Lambda)$ and $|g|$ sufficiently small

$$
\Omega_{\geqq 6} \equiv e^{-W \geqq 6}=\exp \left(-\sum_{Y \subset \Lambda} W_{\geqq 6, Y}\right),
$$

where

$$
\left|W_{\geqq 6, Y}\right| \leqq \mathcal{O}\left(|g|^{1 / 2}\right) e^{-\mu \mathscr{L}(Y)} .
$$

Proof. For $\varphi \in 6 \mathscr{K}(\Lambda)$ and $|g| \leqq \underline{g}$ sufficiently small,

$$
\mathcal{O}(|g|) \sum_{x \in X}\left(\left|\varphi_{x}\right|^{2}+\left|\varphi_{x}\right|^{4}\right) \leqq \mathcal{O}(|g|)|X|\left(L^{N_{0}}\right)^{4} C_{1}^{4} n_{0} \leqq|X| .
$$

Then from Lemma 5 we have $\left|\rho_{\bar{\Delta}}^{\geqq 6}\right| \leqq \mathcal{O}(1)$ and $\left|\rho_{\bar{X}}^{\geqq 6}\right| \leqq \mathcal{O}\left(|g|^{1 / 2}\right) e^{-(\mu / 2) \mathscr{L}(X)}$ for $|X| \geqq 2$. Now $\rho_{\bar{\Delta}}^{\geq 6}$ is analytic in the complex disc $|g| \leqq g$ and the same bound holds there. Since $\rho_{\bar{\Delta}}^{\geqq 6}=1$ at $g=0$ we may estimate the remainder in the Taylor series to get, say for $|g|^{1 / 2} \leqq \underline{g}$

$$
\left|\rho_{\Delta}^{\geqq 6}-1\right| \leqq \mathcal{O}(|g|) \text {. }
$$

Now we write the expansion for $\Omega \geqq 6$ as

$$
\Omega^{\geqq 6}=\prod_{\Delta \subset \Lambda} \rho_{\Delta}^{\geqq 6}\left(\sum_{\left\{X_{\gamma}\right\}} \prod_{\gamma} \tilde{\rho}_{X_{\gamma}}^{\geqq 6}\right),
$$

where the sum is now over all collections $\left\{X_{\gamma}\right\}$ of disjoint subsets $X_{\gamma} \subset \Lambda$ with $\left|X_{\gamma}\right| \geqq 2$, i.e. polymers. We have defined

$$
\tilde{\rho}_{\bar{X}}^{\geqq 6}=\rho_{\bar{X}}^{\geqq 6}\left(\prod_{\Delta \subset X} \rho_{\bar{\Delta}}^{\geqq 6}\right)^{-1} .
$$

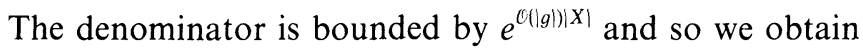

$$
\left|\tilde{\rho}_{\overline{\bar{X}}}{ }^{6}\right| \leqq \mathcal{O}\left(|g|^{1 / 2}\right) \exp (-(\mu / 4) \mathscr{L}(X)) .
$$

From the theory of polymer gases $([5,6,18]$ and earlier references) we have

$$
\sum_{\left\{X_{\gamma}\right\}: X_{\gamma} \subset X} \prod_{\gamma} \tilde{\rho}_{X_{\gamma}}^{\geqq 6}=\exp \left(-\sum_{Y \subset X,|Y| \geqq 2} W_{\geqq 6, Y}\right),
$$


where for $|Y| \geqq 2$,

$$
W_{\geqq 6, Y}=\sum_{n=1}^{\infty} \frac{1}{n !} \sum_{\substack{\left.X_{1}, \ldots, X_{n}\right) \\ \cup X_{i}=Y}} a\left(X_{1}, \ldots, X_{n}\right) \prod_{i=1}^{n} \tilde{\rho}_{\overline{\bar{X}}_{i}}^{\geqq} .
$$

Here the sum is over ordered collections of subsets of $Y$. Each element $X_{i}$ has $\left|X_{i}\right| \geqq 2$, and $a\left(X_{1}, \ldots, X_{n}\right)$ is some coefficient. Using this representation with $X=\Lambda$ and also defining $W_{\bar{\Delta}}^{\geqq 6}=-\left(\log \rho_{\bar{\Delta}}^{\geqq 6}\right)$ we have the claimed representation for $\Omega \geqq 6$.

The function $V_{\geqq 6, Y}$ may be estimated in terms of the quantity

$$
Q \equiv \sup _{\Delta}\left[\sum_{X: \Delta \subset X}\left|\tilde{\rho}_{\bar{X}}^{\geq 6}\right| e^{|X|}\right] \text {. }
$$

By our bound on $\tilde{\rho}_{\bar{X}}^{\geq 6}$ the sum converges and we get $Q \leqq \mathcal{O}\left(|g|^{1 / 2}\right)$. Hence $Q<1$, and so we have the bound (see above references) $\left|W_{\geqq 6, Y}\right| \leqq Q(1-Q)^{-1}|Y| \leqq \mathcal{O}\left(|g|^{1 / 2}\right)|Y|$. This can be improved by first extracting a factor $\exp \left(-(\mu / 8) \mathscr{L}\left(X_{i}\right)\right)$ from each $\tilde{\rho}_{\bar{X}_{i}}^{\geq 6}$ to obtain an overall factor of $\exp (-(\mu / 8) \mathscr{L}(Y))$. Here we use the fact that $a\left(X_{1}, \ldots, X_{n}\right)=0$ if $X_{1}, \ldots, X_{n}$ can be divided into non-overlapping subsets. The rest of the estimate goes as before and we have $\left|W_{\geq 6, Y}\right| \leqq \mathcal{O}\left(|g|^{1 / 2}\right)$ $\exp (-(\mu / 16) \mathscr{L}(Y))$. This is for $|Y| \geqq 2$ but the bound $\left|W_{\geqq 6, \Delta}\right| \leqq \mathcal{O}\left(|g|^{1 / 2}\right)$ for $|Y|=1$ follows from our estimate on $\rho_{\bar{\Delta}}^{\geqq 6}-1$.

Lemma 7. For any $D \subset \Lambda, \varphi \in 2 \mathscr{D}(D, \Lambda)$, and $g$ sufficiently small,

$$
\Omega_{\geqq 6}=\sum_{\left\{X_{j}\right\}}\left(\prod_{j} h_{X_{j}}^{D}\right) \exp \left(-\sum_{Y \subset \sim\left(U_{j} X_{j}\right)} W_{\geqq 6, Y}\right),
$$

where the sum is over collections of disjoint paved sets $X_{j}$ so $D \subset \bigcup_{j} X_{j}$ and so $D \cap X_{j}$ is non-empty union of connected components of D. The quantity $h_{X}^{D}$ depends only on $\varphi \uparrow X$ and satisfies for $C_{2} \geqq \underline{C}_{2}\left(\mu, L, N_{0}\right)$,

$$
\left|h_{X}^{D}\right| \leqq \exp \left((\mathcal{O}|g|)\left(\sum_{x \in D \cap X}\left|\varphi_{x}\right|^{2}+\left|\varphi_{x}\right|^{4}\right)+C_{2}|D \cap X|-\mu \mathscr{L}(X)\right) .
$$

Proof. We begin with the expansion for $\Omega_{\geqq 6}$ in Lemma 5. For each partition $\left\{X_{\delta}\right\}$ let $U$ be the union of the $X_{\delta}$ 's that intersect $D$. Classifying the partitions by $U$ we obtain

$$
\Omega_{\geqq 6}=\sum_{U \supset D}\left[\sum_{\substack{\left\{X_{\delta}\right\} \text { on } U \\ X_{\delta} \cap D \neq \phi}} \prod_{\delta} \rho_{X_{\delta}}^{\geqq 6}\right]\left[\sum_{\left\{X_{\delta}\right\} \text { on } \sim U} \prod_{\delta} \rho_{X_{\delta}}^{\geqq 6}\right] .
$$

The second bracketed expressed is identified as $\exp \left(-\sum_{Y \subset \sim U} V_{\geqq 6, Y}\right)$ since on $\sim D$ the field $\varphi$ is in $6 \mathscr{K}$. Let $\left\{D_{i}\right\}$ be the connected components of $D$. In the first bracketed expression each partition $\left\{X_{\delta}\right\}$ and the $\left\{D_{i}\right\}$ determine a new partition $\left\{X_{j}\right\}$ of $U$ which is the finest such that each $X_{\delta}, D_{i}$ is contained in only one $X_{j}$. Such partitions have the property that $D \cap X_{j}$ is a non-empty union of connected components of $D$. Then the first bracketed expression can be written as a sum over such partitions:

$$
\sum_{\left\{X_{j}\right\} \text { on } U} \prod_{j} h_{X_{j}}^{D},
$$


where

$$
h_{X}^{D}=\sum_{\left\{X_{\delta}\right\} \rightarrow X} \prod_{\delta} \rho_{\bar{X}_{\delta}}^{\geqq 6} .
$$

This last sum is over partitions $\left\{X_{\delta}\right\}$ of $X$ such that $X$ is connected with respect to $\left\{X_{\delta}\right\},\left\{D_{i}\right\}$ (which implies $X_{\delta} \cap D \neq \phi$ ). Combining these observations we obtain the representation of $\Omega_{\geqq 6}$ as stated.

By the estimate on $\rho_{\bar{X}}^{\geqq 6}$ we have

$$
\left|h_{X}^{D}\right| \leqq \exp \left(\mathcal{O}(|g|) \sum_{x \in X}\left(\left|\varphi_{x}\right|^{2}+\left|\varphi_{x}\right|^{4}\right)\right) \sum_{\left\{X_{\delta}\right\} \rightarrow X} \prod_{\delta}\left(\exp \left(-\mu \mathscr{L}\left(X_{\delta}\right)\right)\right) .
$$

Given $\left\{X_{\delta}\right\},\left\{D_{i}\right\}$ connecting $X$ we may form a tree graph connecting $X$ by taking a union of shortest tree graphs on each $X_{\delta}, D_{i}$ and then breaking loops. This gives the estimate $\Sigma_{\delta} \mathscr{L}\left(X_{\delta}\right)+\Sigma_{i} \mathscr{L}\left(D_{i}\right) \geqq \mathscr{L}(X)$. But since $D_{i}$ is connected $\mathscr{L}\left(D_{i}\right) \leqq$ $2^{4} L^{N_{0}}\left|D_{i}\right| \leqq C_{2}\left|D_{i}\right|$. Thus

$$
\sum_{\delta} \mathscr{L}\left(X_{\delta}\right) \geqq-C_{2}|D \cap X|+\mathscr{L}(X),
$$

and so the sum over $\left\{X_{\delta}\right\}$ is bounded by $\exp \left(C_{2}|D \cap X|-(\mu / 2) \mathscr{L}(X)\right)$. Finally since $\varphi$ is bounded on $\sim D$ we obtain $\mathcal{O}(|g|) \sum_{x \in X \sim D}\left(\left|\varphi_{x}\right|^{2}+\left|\varphi_{x}\right|^{4}\right) \leqq|X \sim D| \leqq|X|$ and the factor $e^{|X|}$ is controlled by an $\exp (-(\mu / 4) \mathscr{L} \mathscr{\mathscr { L }}(X))$. Thus we end with a bound of the announced form.

Finally we need a result for $\Omega_{\geqq 4}$.

Lemma 8. For $D \subset \Lambda, \varphi \in 2 \mathscr{D}(D, \Lambda)$, and $g$ sufficiently small,

$$
\Omega_{\geqq 4}=\exp \left(-\delta \lambda_{0} \sum_{x} \varphi_{x}^{4}\right) \sum_{\left\{X_{\omega}\right\}}\left(\prod_{\omega} g_{X_{\omega}}^{D}\right) \exp \left(-\sum_{Y \subset \sim \cup X_{\omega}}\left(\tilde{W}_{4, Y}+W_{\geqq 6, Y}\right)\right),
$$

where the sum is restricted as in Lemma $7, g_{X}^{D}$ only depends on $\varphi \uparrow X$ and

$$
\left|g_{X}^{D}\right| \leqq \exp \left(\mathcal{O}(|g|)\left(\sum_{x \in D \cap X}\left|\varphi_{x}\right|^{2}+\left|\varphi_{x}\right|^{4}\right)+C_{2}|D \cap X|-\mu \mathscr{L}(X)\right) .
$$

Proof. In $\Omega_{\geqq 4}=e^{-W_{4}} \Omega_{\geqq 6}$ we insert $W_{4}=\delta \lambda_{0} \sum_{x} \varphi_{x}^{4}+\tilde{W}_{4}$ and $\Omega_{\geqq 6}$ from Lemma 7. If we also Mayer expand $\exp \left(-\tilde{W}_{4}\right)=\exp \left(-\sum_{Y} \tilde{W}_{4, Y}\right)$ and $\exp \left(-\sum_{Y} W_{\geqq 6, Y}\right)$ we obtain

$$
\exp \left(\delta \lambda_{0} \sum_{x} \varphi_{x}^{4}\right) \Omega_{\geqq 4}=\sum_{\left\{X,\left\{Y_{\alpha}\right\}\left\{Y_{\beta}\right\}\right.} \prod_{j} h_{X_{J}}^{D} \prod_{\alpha}\left(\exp \left(-\tilde{W}_{4, Y_{\alpha}}\right)-1\right) \prod_{\beta}\left(\exp \left(-W_{\geqq 6, Y_{\beta}}\right)-1\right) .
$$

Here $\left\{X_{j}\right\}$ is as in Lemma 7, and the $\left\{Y_{\beta}\right\}$ are restricted by the condition $Y_{\beta} \subset \sim\left(\bigcup_{j} X_{j}\right)$. Each $\left\{X_{j}\right\},\left\{X_{\alpha}\right\},\left\{Y_{\beta}\right\}$ is grouped into clusters $\left\{X_{\omega}\right\}$ which are connected with respect to their constituents. Our sum can now be written

$$
\sum_{\left\{X_{\omega}\right\}} \prod_{\omega} g_{X_{\omega}}^{D},
$$

where the $X_{\omega}$ are disjoint, $D \subset U_{\omega} X_{\omega}$, and $D \cap X_{\omega}$ is a (possibly empty) union of 
connected components of $D$, and where

$$
g_{X}^{D}=\sum_{\{X,\},\left\{Y_{\alpha}\right\},\left\{Y_{\beta}\right\} \rightarrow X} \prod_{j} h_{X_{J}}^{D} \prod_{\alpha}\left(\exp \left(-\tilde{W}_{4, Y_{\alpha}}\right)-1\right) \prod_{\beta}\left(\exp \left(-W_{\geqq 6, Y_{\beta}}\right)-1\right) .
$$

The latter sum is over collections $\left\{X_{j}\right\},\left\{Y_{\alpha}\right\},\left\{Y_{\beta}\right\}$ whose union is $X$ and such that $X$ is connected with respect to them, and so $Y_{\beta} \subset \sim \bigcup_{j} X_{j}$. For each $\left\{X_{\omega}\right\}$ let $U$ be the union of those sets that intersect $D$. Then our sum can be written

$$
\sum_{U \supset D}\left[\sum_{\left\{X_{\omega}\right\} \text { on } U} \prod_{\omega} g_{X_{\omega}}^{D}\right]\left[\sum_{\left\{X_{\omega}\right\} \text { on } \sim U} \prod_{\omega} g_{X_{\omega}}^{D}\right] .
$$

The second sum is over collections $\left\{X_{\omega}\right\}$ of disjoint subset of $\sim U$. For $X_{\omega} \subset \sim U$, $g_{X_{\omega}}$ contains no $X_{j}$ terms and so we may identify this sum as $\exp \left(-\sum_{Y \subset \sim U} \tilde{W}_{4, Y}+W_{\geqq 6, Y}\right)$. The first sum is over partitions of $U$ such that $D \cap X_{\omega}$ is a non-empty union of connected components of $D$. The whole expression now can be rewritten in the stated form.

The bound on $g_{X}^{D}$ follows as in the previous lemmas; see particularly Lemma 5.

\section{Conclusions}

We now collect our results. The new field strength in $\mathscr{H}_{0}(\varphi)$ is identified as $Z_{0}=$ $1+\zeta_{0}\left(\zeta_{0}\right.$ from Lemma 2$)$ and we renormalize to one by considering $\mathscr{H}_{0}\left(Z_{0}^{-1 / 2} \varphi\right)$.

Theorem. Given $M$ let $L, N_{0}, C_{0}, C_{2}, C_{1}, n_{0}$ be sufficiently large and chosen in the indicated order. Let $C_{-} n_{0}^{-1}<\lambda_{0}<C_{+} n_{0}^{-1}$ and $m_{0}^{2} \in\left(-n_{0}^{-3 / 2}, n_{0}^{-3 / 2}\right)$. Then for $g$ sufficiently small $\mathscr{H}_{0}\left(Z_{0}^{-1 / 2} \varphi\right)$ satisfies GKI, GKII.

Proof. Given $M$, choose $\mu>0$ sufficiently small, and then $L, N_{0}$ large so our lemmas hold. We may assume also that $\alpha \leqq \mu$ (since $\alpha=\alpha(L)=\mathcal{O}\left(L^{-1}\right)$ ) and hence $e^{-\mu \mathscr{L}(Y)} \leqq e^{-\alpha \mathscr{L}(Y)}$.

To verify GKI suppose $\varphi$ is in the small field region. We insert $-\log \Omega=$ $W_{2}+W_{4}+W_{\geqq 6}$ in $\mathscr{H}_{0}$ and then we have

$$
\mathscr{H}_{0}\left(Z_{0}^{-1 / 2} \varphi\right)=\frac{1}{2} \sum_{x, y} \varphi_{x}\left(G_{0}^{-1}\right)_{x y} \varphi_{y}+V(\varphi)
$$

where now

$$
G_{0}^{-1}=G_{0}^{-1}\left(Z_{0}^{-1} \xi\right),
$$

and where

$$
\begin{aligned}
V_{2}(\varphi) & =\sum_{x} \varphi_{x}^{2}\left(\frac{1}{2}\left(m_{0}^{\prime}\right)^{2}-6 \lambda_{0}^{\prime} G_{0, x x}\right)+\sum_{\mu, v x, y} \sum_{\mu}\left(\partial_{\mu} \varphi\right)_{x} K_{\mu \nu}^{\prime}(x, y)\left(\left(\partial_{\nu} \varphi\right)_{y}-\left(\partial_{\nu} \varphi\right)_{x}\right), \\
V_{4}(\varphi) & =\lambda_{0}^{\prime} \sum_{x} \varphi_{x}^{4}+\sum_{Y} \tilde{W}_{4, Y}\left(Z_{0}^{-1 / 2} \varphi\right), \\
V_{\geqq 6}(\varphi) & =\sum_{Y} W_{\geqq 6, Y}\left(Z_{0}^{-1 / 2} \varphi\right),
\end{aligned}
$$


and where

$$
\begin{aligned}
K_{\mu \nu}^{\prime}(x, y) & =Z_{0}^{-1} K_{\mu \nu}(x, y) \\
\left(m_{0}^{\prime}\right)^{2} & =Z_{0}^{-1}\left(m_{0}^{2}+\delta m_{0}^{2}\right)+12\left(\lambda_{0}^{\prime} G_{0, x x}\left(Z_{0}^{-1} \xi\right)-\lambda_{0} Z_{0}^{-1} G_{0, x x}(\xi)\right) \\
\lambda_{0}^{\prime} & =Z_{0}^{-2}\left(\lambda_{0}+\delta \lambda_{0}\right) .
\end{aligned}
$$

It is now easy to check the various conditions. Since $Z_{0}=1+\mathcal{O}\left(g^{2}\right), \delta m_{0}^{2}=$ $\mathcal{O}\left(g^{2}\right), \delta \lambda_{0}=\mathcal{O}\left(g^{4}\right)$, we have $\lambda_{0}^{\prime},\left(m_{0}^{\prime}\right)^{2}$ in the same intervals as $\lambda_{0}, m_{0}^{2}$ for $g$ small and hence GKI(a), GKI(b). Condition GKI(c) for $K^{\prime}$ follows from the bound on $K$ in Lemma 2. For GKI(d) we use the expression for $\tilde{W}_{4, Y}$ in the remark after Lemma 3. Assuming $\left|\varphi_{i, x}\right| \leqq C_{1} n_{0}^{1 / 4}$ for $i=1,2,3$ and $\left|\varphi_{4, x}-\varphi_{4, y}\right| \leqq$ $C_{0} C_{1} n_{0}^{1 / 4}|x-y|$ and using the bound on $\Lambda_{Y}$ we obtain the required bound for $\tilde{W}_{4, Y}\left(Z_{0}^{-1 / 2} \varphi\right)$. Finally if $\varphi \in 3 \mathscr{K}(\Lambda)$ we have $Z_{0}^{-1 / 2} \varphi \in 6 \mathscr{K}(\Lambda)$ and the bound GKI(e) for $W_{\geq 6, Y}\left(Z_{0}^{-1 / 2} \varphi\right)$ follows by Lemma 6 .

Now for GKII suppose $\varphi \in \mathscr{D}(D, X)$. We have by Lemma 8

$$
\begin{gathered}
\left(e^{-V_{\geqq 4}}\right)(\varphi) \equiv e^{V_{2}(\varphi)}\left(e^{-V}\right)(\varphi)=\exp \left(-\lambda_{0} Z_{0}^{-2} \sum_{x} \varphi_{x}^{4}\right) \Omega_{\geqq 4}\left(Z_{0}^{-1 / 2} \varphi\right) \\
=\sum_{\left\{X_{\omega}\right\}} \prod_{\omega}\left(g^{\prime}\right)_{X_{\omega}}^{D} \cdot \exp \left(-\lambda_{0}^{\prime} \sum_{x \in \sim D} \varphi_{x}^{4}-\sum_{Y \subset \sim \bigcup X_{\omega}}\left(\tilde{W}_{4, Y}\left(Z_{0}^{-1 / 2} \varphi\right)\right)+W_{\geqq 6, Y}\left(Z_{0}^{-1 / 2} \varphi\right)\right),
\end{gathered}
$$

where

$$
\left(g^{\prime}\right)_{X}^{D}(\varphi)=\exp \left(-\lambda_{0}^{\prime} \sum_{x \in X \cap D} \varphi_{x}^{4}\right) g_{X}^{D}\left(Z_{0}^{-1 / 2} \varphi\right)
$$

We have the estimate for $C_{2}$ large enough (as in [12], p. 222)

$$
\begin{aligned}
& \left|\exp \left(-\lambda_{0}^{\prime} \sum_{x \in D \cap X} \varphi_{x}^{4}\right)\right| \leqq \exp \left(-\lambda_{0}^{\prime} / 4 \sum_{x \in D \cap X}\left(\operatorname{Re} \varphi_{x}\right)^{4}\right) \\
& \cdot \exp \left(\frac{1}{2} C_{2}|D \cap X|+\sum_{x \in D \cap X}\left(-\left(\lambda_{0}^{\prime}\right)^{1 / 2}\left|\varphi_{x}\right|^{2}+18 \lambda_{0}^{\prime}\left(\operatorname{Im} \varphi_{x}\right)^{4}\right) .\right.
\end{aligned}
$$

On the other hand since $Z_{0}^{-1 / 2} \varphi \in 2 \mathscr{D}(D, x)$ we have by Lemma 8

$$
\left|g_{X}^{D}\left(Z_{0}^{-1 / 2} \varphi\right)\right| \leqq \exp \left(\mathcal{O}(|g|) \sum_{x \in D \cap X}\left(\left(\operatorname{Re} \varphi_{x}\right)^{4}+\left(\operatorname{Im} \varphi_{x}\right)^{4}\right)+\frac{1}{2} C_{2}|D \cap X|-\alpha \mathscr{L}(X)\right) .
$$

Provided then that $\mathcal{O}(|g|)<\lambda_{0}^{\prime} / 4$ the $\left(\operatorname{Re} \varphi_{x}\right)^{4}$ term has a negative coefficient and we obtain a bound of the required form on $\left(g^{\prime}\right)_{X}^{D}$. The identity for $\left(g^{\prime}\right)_{X}^{D}$ can also be checked and so GKII is established.

Remark. Since $m_{0}^{2}$ is unrestricted we can actually obtain any value for $\left(m_{0}^{\prime}\right)^{2}$ in $\left(-n_{0}^{-3 / 2}, n_{0}^{-3 / 2}\right)$ by adjusting $m_{0}^{2}$.

Corollary. Fix $M$ and let $\lambda_{0} \leqq \underline{\lambda}_{0}, g \leqq g\left(\lambda_{0}\right)$ be sufficiently small. Then one can choose $m_{0}^{2}=m_{0}^{2}\left(\lambda_{0}, g\right)$ such that $\mathscr{H}_{0}\left(Z_{0}^{-1 / 2} \varphi\right)$ is asymptotically free under the flow of the renormalization group. The infinite volume limit exists for the correlation functions (for either $\mathscr{H}_{0}\left(Z_{0}^{-1 / 2} \varphi\right)$ or $\mathscr{H}_{0}(\varphi)$ ) and they define a critical theory.

Proof. We assume $\lambda_{0}$ is sufficiently small so that $\lambda_{0}<C_{+} n_{0}^{-1}$ for the $n_{0}$ of the theorem. Then by taking $n_{0}$ even larger if necessary we can arrange $C_{-} n_{0}^{-1}<\lambda_{0}<C_{+} n_{0}^{-1}$. Then GKI, GKII hold for $g$ sufficiently small by the 
theorem. Now choose $m_{0}^{2}$ so that $\left(m_{0}^{\prime}\right)^{2}=f\left(\lambda_{0}^{\prime}\right)$ and the conclusions follow from the general results of Gawedzki-Kupiainen as explained in the introduction.

Remarks.

(1) It would be interesting to keep the fermions as dynamical variables and try to adjust parameters so that both the boson mass and the fermion mass vanish. This could lead to a treatment of the continuum limit except that theory is not asymptotically free in the UV. One would have to make some modification such as negative $\lambda_{0}[13]$ or (for the future) adding a non-abelian gauge field.

(2) Another interesting variation would be to study infrared properties for (QED) ${ }_{4}$ on a lattice. For this problem one would expect that gauge invariance would guarantee a vanishing mass, and one could dispense with any adjustments of the bare mass. On the other hand one would not have a $\varphi^{4}$ term to stabilize the theory which could make life more difficult.

\section{References}

1. Balaban, T.: Recent results in constructing gauge fields. Physica 124A, 79-90 (1984)

2. Balaban, T.: Renormalization group approach to lattice gauge field theories. Harvard preprint

3. Balaban, T., Gawedzki, K.: A low-temperature expansion for the pseudoscalar Yukawa model of quantum fields in two space-time dimensions. Ann. Inst. H. Poincaré 36, 271-400 (1982)

4. Balaban, T., Imbrie, J., Jaffe, A.: Exact renormalization group for gauge theories. In Progress in gauge theories. pp. 79-104, 1983 Cargese Lectures, Lehmann, H., t'Hooft, G. Jaffe, A., Mitter, P., Singer, I., Stora, R. (eds.) New York: Plenum Press 1984

5. Brydges, D.: A short course on cluster expansions. 1984 Les Houches lectures (to appear)

6. Cammarota, C.: Decay of correlations for infinite range interactions in unbounded spin systems. Commun. Math. Phys. 85, 517-528 (1982)

7. Feldman, J., Magnen, J., Rivasseau, V., Sénéor, R.: The infrared behavior of $\phi_{4}^{4}$, Ecole Polytechnique preprint

8. Feldman, J., Magnen, J., Rivasseau, V., Sénéor, R.: A renormalizable field theory: The massive GrossNeveu model in two dimensions. Commun. Math. Phys. 103, 67-103 (1986)

9. Federbush, P.: A phase cell approach to Yang-Mills theory. Michigan preprints

10. Gawedzki, K., Kupiainen, A.: A rigorous block spin approach to massless lattice theories. Commun. Math. Phys. 77, 31-64 (1980)

11. Gawedzki, K., Kupiainen, A.: Block spin renormalization group for dipole gas and $(\nabla \varphi)^{4}$. Ann. Phys. 147, 198-243 (1983)

12. Gawedzki, K., Kupiainen, A.: Massless lattice $\phi_{4}^{4}$ theory: Rigorous control of a renormalizable asymptotically free model. Commun. Math. Phys. 99, 197-252 (1985)

13. Gawedzki, K., Kupiainen, A.: 1984 Les Houches lectures (to appear)

14. Gawedzki, K., Kupiainen, A.: Gross-Neveu model through convergent perturbation expansions. Commun. Math. Phys. 102, 1-30 (1985)

15. Glimm, J., Jaffe, A., Spencer, T.: In Constructive quantum field theory. pp. 132-242. Velo, G. Wightman, A. (eds.), Springer Berlin, Heidelberg, New York:

16. Mathews, P., Salam, A.: The renormalization of meson theories. Rev. Mod. Phys. 23, 311-314 (1951)

17. Seiler, E.: Schwinger functions for the Yukawa model in two-dimensions with space-time cutoff. Commun. Math. Phys. 42, 163-182 (1975)

18. Seiler, E.: Gauge Theories as a problem of constructive quantum field theory and statistical mechanics. Lecture Notes in Physics Vol. 159, Berlin, Heidelberg, New York: Springer 1982.

19. Simon, B.: Notes on infinite determinants of Hilbert space operators. Adv. Math. 24, 244-273 (1977)

Communicated by K. Osterwalder

Received June 11, 1986 
